Published in final edited form as:

Immunol Rev. 2014 January ; 257(1): 237-249. doi:10.1111/imr.12128.

\title{
Hematopoietic Stem Cells for Cancer Immunotherapy
}

\author{
Eric Gschweng ${ }^{1,2}$, Satiro De Oliveira ${ }^{3,4}$, and Donald B. Kohn ${ }^{1,2,3,4}$ \\ ${ }^{1}$ Department of Microbiology, Immunology and Molecular Genetics, University of California, Los \\ Angeles, David Geffen School of Medicine, Los Angeles, CA, USA
}

${ }^{2}$ Eli and Edythe Broad Center for Regenerative Medicine \& Stem Cell Research University of California, Los Angeles, David Geffen School of Medicine, Los Angeles, CA, USA

${ }^{3}$ Department of Pediatrics, University of California, Los Angeles, David Geffen School of Medicine, Los Angeles, CA, USA

${ }^{4}$ The Jonsson Comprehensive Cancer Center, University of California, Los Angeles, David Geffen School of Medicine, Los Angeles, CA, USA

\section{Abstract}

Summary: Hematopoietic stem cells (HSCs) provide an attractive target for immunotherapy of cancer and leukemia by the introduction of genes encoding T-cell receptors (TCR) or chimeric antigen receptors (CARs) directed against tumor-associated antigens. HSCs engraft for long-term blood cell production and could provide a sustained source of targeted anti-cancer effector cells to sustain remissions. T cells produced de novo from HSCs may continuously replenish anti-tumor T cells that have become anergic or exhausted from ex vivo expansion or exposure to the intratumoral microenvironment. Additionally, transgenic $\mathrm{T}$ cells produced in vivo undergo allelic exclusion, preventing co-expression of an endogenous TCR that could mis-pair with the introduced TCR chains and blunt activity or even cause off-target reactivity. CAR-engineered HSCs may produce myeloid and NK cells in addition to T cells expressing the CAR, providing broader anti-tumor activity that arises quickly after transplant and does not solely require de novo thymopoiesis. Use of TCR- or CAR-engineered HSC would likely require cytoreductive conditioning to achieve long-term engraftment, and this approach may be used in clinical settings where autologous HSC transplant is being performed to add a graft-versus-tumor effect. Results of experimental and pre-clinical studies performed to date are reviewed.

\section{Keywords}

hematopoietic stem cells; immunotherapy; T-cell receptor; chimeric antigen receptor

\section{T cells for TCR- and CAR-mediated immunotherapy: progress and limitations}

Studies of immunotherapy using peripheral blood T lymphocytes modified with genes encoding T-cell receptors (TCRs) or chimeric antigen receptors (CARs) recognizing tumorassociated antigens have made great strides in our understanding of basic immunology and yielded excellent clinical responses in some settings (as discussed elsewhere in this issue). Pioneering work by Steven Rosenberg at the Surgery Branch of the National Cancer Institute $(1,2)$ paved the way for a plethora of studies that have improved the efficacy of this

Correspondence to: Donald B. Kohn, M.D. 3163 Terasaki Life Science Bldg. 610 Charles E. Young Drive Los Angeles CA 91356 Tel.: +1 3107941964 Fax: +1 3102060356 dkohn@mednet.ucla.edu.

The authors have no financial conflict of interest with the information presented here. 
therapeutic approach utilizing ex vivo expanded antigen-specific tumor infiltrating lymphocytes reinfused into patients (1), as well as the engineering of peripheral blood T cells via viral gene transfer (2). Further work using CARs has recently shown great promise in several trials: engineering $\mathrm{T}$ lymphocytes with CARs directed against the B-lymphocyte antigen CD19 has led to complete remissions in a number of patients with advanced Blineage malignancies (3-6). The potential of these therapies is indisputable, though limitations remain. Objective responses using TCR engineered T cells have been achieved in the majority of patients enrolled in trials: a measurable decrease in bulk tumor burden is often observed. However, the complete response rates have been quite low and the majority of clinical responses are short-lived with ultimate tumor relapse. This has been observed particularly with TCR-based approaches, though CAR-based approaches may also have some sub-optimal aspects as further studies are reported.

A major explanation for this sub-optimal outcome is the relatively limited in vivo survival or suppression or exhaustion of infused engineered T cells $(7,8)$. Expansion of T cells to an effective therapeutic bolus is achieved by ex vivo culture in supraphysiologic concentrations of IL-2, increasing the cell numbers by several orders of magnitude. It has been appreciated that driving cells to expand under these conditions ages the cells from a more naive and replicative phenotype to late-stage effectors. Characterized by the loss of markers CD45RA, CD62L, and CCR7, these cells have great cytotoxic capacity but greatly diminished regenerative potential. Experimental work in murine models (9) as well as non-human primates (10) has shown the improved anti-tumor efficacy of central memory phenotype cells $(\mathrm{Tcm})$ versus late stage effectors (9). There are promising recent reports showing that including small molecule inhibitors or modulation of the cytokine milieu in which cells are expanded ex vivo makes possible the maintenance and generation of the more stem like Tcell populations known as stem cell memory (Tscm) cells, and that these cells are capable of a more sustained response by replenishing effectors (11) similar to the previous studies with Tcm cells. A clear benefit to the transfer of less mature, more stem-like cells is evident, likely due to the increased persistence and replenishing capability of these cells in vivo.

In accordance with this hypothesis, studies investigating T cells transduced with CARs seem to have greater persistence and activity than those modified with TCRs, potentially demonstrating improved efficacy. Scholler et al. (12) reported long-term follow-up of subjects who received $\mathrm{T}$ cells modified to express a CAR-like molecule targeted against HIV-infected cells, and even 10-15 years after a single infusion of modified T cells, these cells could still be detected in the peripheral blood of many subjects. Recent trials using CARs against CD19 have shown significant clinical efficacy and also observed persistence of the engineered T cells, although with a shorter follow-up time (3-6). The inclusion of costimulatory domains derived from CD28 or 4-1BB may lead to low level tonic signaling that sustains T-cell survival in vivo; alternatively, chronic exposure to the CD19 antigen as new B-cell precursors are produced in the marrow may induce survival of the CARmodified cells (13).

Aside from the reduced persistence and activity, another theoretical concern for the use of mature T cells as targets for anti-cancer TCRs is the potential for mis-pairing: that is, the pairing of endogenous cellular TCR chains with introduced transgenic chains. In this situation, T cells can be redirected, miss the intended cancer-antigen target, and instead be reactive to unpredictable moieties expressed on normal tissue. This has been demonstrated in a C57/B16 murine based study of immunotherapy using TCR $\alpha$ - and $\beta$-chains to engineer $\mathrm{T}$ cells via adoptive transfer (14). This system mimicked the clinical setting by including total body irradiation (TBI) and IL-2, and examined a variety of commonly studied TCR including OT-I, gp100, MART-1, and pmel-1. It was clearly demonstrated that mice developed symptoms of graft versus host disease (GvHD), and that the introduced T cell 
chains, as a pair or individually, were necessary and sufficient to cause this outcome independent of IL-2 administration. It is important to note that the development of GvHD as a result of mis-pairing of an exogenous TCR introduced into mature T cells with an endogenous TCR chain has not been an observed problem in any clinical trial to date (15), though it remains a theoretical possibility.

The real or artifactual potential for induction of frank autoimmunity aside, the reduction of desired antigen-specific TCR surface expression due to mis-pairing poses another hurdle. A fraction of the transgenic TCR chains may be tied-up in pairs with endogenous TCR chains that do not possess the desired specificity, blunting their effectiveness for anti-tumorspecific activity by reducing the surface density of engineered TCR expression. As the surface density of TCR is correlated with activation $(16,17)$, the reduction of cancer antigen-specific TCR may render engineered cells incapable of mounting an effective immune response, nullifying therapeutic benefit. A number of approaches to reduce or eliminate the possibility of TCR mis-pairing have been described (18, reviewed elsewhere in this volume).

\section{Hematopoietic stem cells for TCR- and CAR-mediated immunotherapy}

Approaching T-cell immunotherapy by engineering of autologous hematopoietic stem cells (HSCs) with a pre-arranged anti-cancer-antigen TCR or CAR may offer a one-two punch, solving both the problem of effector cell persistence and TCR mis-pairing at the same time. HSCs are the most primitive of blood lineage cells. Residing predominantly in the bone marrow, HSCs produce all mature blood cells by proliferation and differentiation through a series of increasingly lineage-restricted progenitors. HSC transplantation has been a clinical practice for more than four decades to promote life-long hematological recovery after high doses of chemotherapy or radiation to treat malignancies, for marrow reconstitution in marrow failure states, such as aplastic anemia, and to treat genetic diseases of blood cells, by providing a source of normal stem cells that can produce the needed normal blood cell type. HSCs can be obtained for transplant from the bone marrow of a suitably matched donor, by leukopheresis of peripheral blood after mobilization by administration of pharmacological doses of cytokines such as G-CSF [peripheral blood stem cells (PBSCs)], or from the umbilical cord blood (UCB) collected from the placenta after delivery. The marrow, PBSCs, or UCB may be transplanted without processing, or the HSCs may be enriched by immuneselection with a monoclonal antibody to the CD34 surface antigen, especially if the HSC are to be modified by ex vivo gene transfer. Further enrichment of HSCs is an active area of research $(19,20)$ that will no doubt improve subsequent gene therapeutic approaches to immunotherapy.

Gene transfer and expression in HSCs has been under study for more than three decades (21). Vectors derived from viruses of the Retroviridae family, either $\gamma$-retroviral, such as murine leukemia viruses (MLV), lentiviral, such as the human immunodeficiency virus (HIV), or spumaviral, such as the human foamy virus (HFV), have been most effective for permanent gene insertion into the chromosomes of HSCs. This results in stable transmission to all progeny progenitors and mature blood cells. Typical clinical approaches to gene transduction of HSCs involves enrichment for the $\mathrm{CD} 34^{+} \mathrm{T}$-cell fraction, culture in medium containing a cocktail of recombinant human cytokines including c-kit ligand, flt-3 ligand, and thrombopoietin to activate or pre-stimulate the HSC from quiescence for 1-2 days, and then exposure to the gene delivery vector for 1-2 days by its addition to the culture. At the completion of transduction, the cells are washed and formulated for either direct intravenous infusion or cryopreservation for transplantation at a later date. Using these current optimized methods, insertion of 1-3 copies of the delivered transgene may be achieved into the 
majority of the HSCs, with preservation of their engraftment and multi-potent blood cell production capacity.

Expression of the delivered transgene is typically achieved by the use of a strong constitutive transcriptional control element such as a viral or cellular enhancer/promoter fragment. The transgene product may be transcribed in blood cells of all lineages produced from the HSCs, although other factors may modulate their expression. For example, the requirement for co-expression of the CD3 complex of proteins limits surface display of a transgenic TCR to T lymphocytes, even though the vector may be present and transcribed in all blood cells derived from a transduced HSC (notably, CARs are not limited by CD3 expression). Alternatively, some vectors are designed to use lineage-specific transcriptional control elements to restrict expression to specific cell types, such as erythroid, myeloid, etc.

The approach of using HSCs as the targets for TCRs or CARs is attractive for at least three reasons. First, as with approaches that use Tcm or Tscm as targets for gene modification, the regenerative nature of HSCs may provide a long lasting (potentially life-long) supply of effector T cells engineered against the antigen of interest. TCR- or CAR-modified HSCs will continuously produce T-lymphocyte progenitors that will undergo normal thymopoiesis and development, increasing the potential for development of immunological memory (Fig. 1). This is in contrast to mature T cells massively expanded ex vivo before infusion, with most having a finite life-span. Second, the off-target cytotoxicity or blunted surface expression of the engineered TCRs due to mis-pairing described above as a potential complication from transducing mature $\mathrm{T}$ cells may be eliminated by tackling the approach from HSCs that have not yet rearranged their germline TCR loci (22) if the transgenic TCR suppresses rearrangement of the endogenous TCR loci. This allelic exclusion would ensure the robust surface expression of an exclusive, antigen-targeted TCR. Third, it has been demonstrated that increasing the intensity of the pre-conditioning regimens used when administering tumor-infiltrating lymphocytes correlates with increasing clinical efficacy (23). Very high doses of conditioning (essentially cytoablative) mandate HSCs rescue of the patient, which provides an ideal setting for engineering a portion of the HSC graft for immunotherapy.

In this review, we discuss the known benefits and potential pitfalls associated with using HSCs for engineered immunotherapy and recent work focused on moving this technology to a clinical setting.

\section{TCRs into HSCs: Imperium sine fine dedi}

Stable retroviral or lentiviral introduction of pre-arranged cancer antigen-specific TCR or CAR to HSCs can provide an ongoing source of targeted T cells and, perhaps, other effectors. While infusions of TCR- or CAR-modified mature T cells have an anti-neoplastic impact within days to weeks, production of anti-tumor effector cells from engineered, transplanted HSCs may take longer, with myeloid and NK cells emerging within 1-3 weeks after transplantation and de novo T lymphopoiesis occurring only after several months. Though delayed, it is hypothesized that the stable engraftment of TCR or CAR-modified HSCs will lead to sustained production of targeted effector cells, potentially providing sustained anti-tumor activity.

It is well established that the transduction and transplant of HSC with a prearranged TCR can give rise to antigen-specific T cells in murine models. Early reports $(24,25)$ used retroviral vectors to deliver TCRa and TCR $\beta$ chains to mouse bone marrow followed by transplantation into lethally irradiated recipients. Long term development of $\mathrm{T}$ cells expressing the introduced TCR was demonstrated, including in secondary recipients, 
providing evidence of gene transfer to long-term HSCs. Later work showed that engineered T cells produced from TCR gene-transduced murine HSCs that were transplanted into lethally irradiated hosts were responsive to antigen stimulation by dendritic cells, developed a memory cell phenotype, and provided protection against in vivo tumor challenge $(26,27)$. Alajez et al. (28) used a novel fusion protein consisting of a single chain TCR that recognized a segment of the MUC1 tumor-associated protein in a non-MHC-restricted manner with the $\mathrm{CD} 3 \zeta$ chain intracellular signaling domain (in effect a chimeric antigen receptor) to transduce murine bone marrow which was transplanted into SCID mice. The recipients of the fusion protein-transduced marrow rejected MUC1 positive tumors more effectively than did controls.

Two studies examined the potential to produce T cells from transduced human HSCs using in vitro differentiation on the murine OP9-DL1 stromal cell line. Zhao et al. (29) transduced human UCB with retroviral vectors encoding TCR against relevant human tumor associated antigen peptides (NY-ESO-1 and p53) and directed their differentiation to T lymphocytes by co-culture on OP9-DL1 stromal cells. The T cells so produced displayed antigen-specific, HLA-restricted cytolytic activity. Van Lent et al. (30) used retroviral vectors to introduce the cDNA for human TCR $\alpha$ and TCR $\beta$ chains recognizing peptides from either HA-2, CMV, or MART-1 into either human thymic T-lymphoid progenitor cells $\left(\mathrm{CD} 34^{+} \mathrm{CD} 1 \mathrm{a}^{-}\right)$or human UCB $\left(\mathrm{CD} 34^{+} \mathrm{CD} 38\right)$ progenitor/stem cells. Mature T cells were produced in vitro on OP9-DL1 stroma that possessed cytolytic activity with the antigen specificity of the introduced TCR. Interestingly, the introduction of only a TCR $\beta$ chain greatly accelerated T lymphopoiesis, postulated to result from prolonged signaling from a preTCR complex formed with a pTa chain, whereas introduction of both the TCR $\beta$ and a chains only moderately increased T-cell production, compared to cultures receiving only the TCRa chain or control non-transduced cultures.

TCR engineering of human HSCs to produce modified T cells in vivo was first shown using a model in which human thymic tissue is implanted into NOD/SCID/IL2 $\mathrm{Rg}^{-/-}$(NSG) immune-deficient mice, followed by injection of gene-modified human $\mathrm{CD}^{+} 4^{+}$cells (the BLT model). This study demonstrated the development of anti-HIV T cells after transfer of the genes encoding a TCR against an HIV-1 gag peptide. Functional, antigen-specific T effector cells were produced that were capable of killing HIV-infected cells (31). This same model was then used to generate MART-1 antigen-reactive human T-cell effectors capable of killing melanoma tumors in an antigen- and HLA-specific context after transplantation of human fetal liver-derived CD34+ ${ }^{+}$HSCs transduced with the genes encoding a TCR to the melanocyte antigen MART-1 (32). One caveat to the BLT model is that the education of T cells in a human thymic organoid does not induce tolerance to the mouse host, generating self-reactive $T$ cells that ultimately kill the humanized mouse via GvHD, limiting the duration of studies to short-term (33). Though efficacy can be demonstrated in this model in an antigen- and HLA-specific context, the examination of long-term provision of cells is hampered.

To address this issue, we used a transplant model where the clinically relevant human HSC sources of CD34-enriched UCB or PBSC are transplanted into sub-lethally irradiated NSG immune-deficient neonatal mice (Fig. 2). In a direct human HSC transplant to NSG neonates, developing human thymocytes are educated on the murine thymus. While this likely results in a reduced TCR repertoire compared with the BLT model, the mature T cells are host-tolerant, allowing long-term studies of these chimeric animals. Furthermore, using NSG hosts engineered to express human MHC molecules can allow the development of an HLA-restricted T-cell repertoire (34). 
In NSG and NSG-HLA*A0201 mouse strains, we studied the development of human HSCs transduced with a TCR recognizing the $27-35$ MART-1 nonamer. We demonstrated that both $\mathrm{CD} 8^{+}$and $\mathrm{CD}^{+}{ }^{+}$cells were produced that expressed the introduced TCR (22). The presence of the pre-arranged TCR in the developing thymocytes appeared to accelerate and boost $\mathrm{CD}^{+} \mathrm{T}$-cell production, as had been observed in vitro by van Lent et al. (30). The $\mathrm{T}$ cells produced showed antigen-specific release of $\gamma$-interferon when tested ex vivo (22). Though the body of literature describing engineered immunity via HSCs is relatively young, proof of principle has been demonstrated showing the promise of this approach. Further work to classify the T-cell phenotypes developing in this model, and exploration of mouse models that mimic the human cytokine milieu and how they affect T-cell development and homeostasis in the context of engineered immunity via HSCs remain interesting arenas for exploration.

In addition to the potential life-long provision of effector cells, the engineering of HSCs with exogenous TCRs is an attractive solution to the issue of TCR mis-pairing. While inclusion of a number of clever designs to the TCR construct can minimize this undesired consequence, the HSC approach is capable of sidestepping this pitfall entirely due to the mechanism of allelic exclusion. Early in T-cell development, the endogenous TCR $\beta$ and TCRa genes undergo V(D)J rearrangement via the VDJ recombination complex of DNA modifying enzymes. Upon successful rearrangement of one allele, the recombination of the remaining germ-line allele is inhibited (at least for TCR $\beta$ ). This ensures that only a single $a$ and single $\beta$ chain will be expressed in a given T cell, conferring a single MHC-specific antigenic reactivity (the same allelic exclusion mechanism is present in B cells, where it was discovered) (35).

Work by our group at UCLA has demonstrated genetically (22) and molecularly (36) that the expression of a prearranged TCR upstream of T-cell development by the engineering of human HSCs does indeed induce allelic exclusion as in normal development. Deep sequencing of the TCRV $\beta$ CDR3 region in T cells derived from HSCs engineered to express a transgenic TCR specific for the $27-35$ MART-1 peptide and differentiated into T cells in the NSG mouse showed only the introduced T-cell rearrangement, whereas T cells lacking expression of the introduced TCR contained a broad array of TCRV $\beta$ gene rearrangements (22). In line with this finding, quantitative analysis of TREC, RNA spectratyping, and TCRV $\beta$ chain expression by flow cytometry demonstrated allelic exclusion in MART- $1^{+} \mathrm{T}$ cells when compared to MART-1 ${ }^{-}$T cells in a BLT model (36). In the latter study, while V $\beta$ family member surface staining clearly demonstrated exclusion, spectratyping of RNA showed enrichment, but not exclusive expression, of the transgenic TCR chain. This result may suggest leakiness of allelic exclusion in this model or perhaps post-transcriptional silencing of only one rearranged TCR loci resulting in surface expression an exclusive V $\beta$ chain. These somewhat contradictory findings may highlight differences in HSC progenitors from fetal liver versus UCB, or of the thymopoietic processes in BLT versus the NSG models; a comprehensive study examining engineered immunity induced allelic exclusion at the DNA, RNA, and protein levels would be informative.

More recently, we have performed studies using a lentiviral vector carrying the cDNA for a human TCR recognizing the 157-165 peptide from the NY-ESO-1 tumor-associated antigen (37). This work has focused on the use of human PBSC as a clinically relevant HSC source compared to fetal liver or UCB, as PBSCs will be the cell source used for an upcoming trial of HSC based immunotherapy at UCLA. While higher doses of CD34 $4^{+} \mathrm{PBSCs}$ are needed to effectively engraft the NSG mice compared to what was used with UCB $\left(1 \times 10^{6}\right.$ versus $1-3 \times 10^{5}$, respectively), we have observed the production of T cells expressing the anti-NYESO-1 TCR in mice transplanted with transduced PBSCs. Extensive immunophenotypic characterization of T cells produced in vivo and expressing the TCR transgene is in progress. 
In contrast to the TCR against MART- 1 that appeared on both $\mathrm{CD}^{+}$and $\mathrm{CD} 8^{+} \mathrm{T}$ cells, expression of the NY-ESO-1 TCR has been restricted to $\mathrm{CD}^{+}+\mathrm{T}$ cells, indicating that the specific TCR plays an instructive role in T-cell development (Fig. 3).

The NY-ESO-1 TCR lentiviral vector also carries the modified herpes simplex thymidine kinase gene (sr39TK) that functions as both a PET reporter as well as a potential suicide gene (see below) (38). The transferred TCR proteins will only be displayed on the surface of $\mathrm{T}$ cells produced from the transduced HSCs due to the requirement for co-expression of CD3, limiting detection of total gene modified cells using a TCR assay based approach such as binding to MHC-tetramer. However, the sr39TK reporter gene will be expressed at high levels from the vector's MSCV LTR promoter in cells of all hematopoietic lineages. This reporter allows in vivo imaging to be performed to examine sites of hematopoiesis, visualizing the gene-modified progeny of the transduced $\mathrm{CD} 34^{+}$cells in vivo in the NSG recipients. Following administration of an $\left[{ }^{18} \mathrm{~F}\right]$-FHBG PET probe $(200-250 \mathrm{uCi})$ to the mice, we have observed hematopoietic niches including the long bones, sternum, spleen, vertebrae, ribs, as well as the thymus as hot spots of reporter signal, indicative of engrafted, transduced human HSCs and their cell progeny in NSG hosts (E.G., manuscript in preparation).

As central tolerance limits the development of self-reactive $\mathrm{T}$ cells, and cancer antigens targeted in T-cell therapy are frequently self-expressed antigens (39). As such, the isolation of self-reactive TCRs is quite rare and, when present, the TCR affinity is accordingly low (40). This principle potentially poses a challenge for the TCR-modified HSC approach. Mature T cells have already passed the stage of development where auto-reactive cells have been deleted, and the introduction of a self-reactive TCR into these cells is possible. However, in a pre-thymic stem/progenitor cell destined for thymic education and surveillance, auto-reactivity may result in deletion of thymocytes expressing self-reactive TCR.

An elegant approach to circumvent this limitation has been demonstrated in the mouse (41). In this study, the investigators determined that microRNA miR-181 family members were expressed transiently at high levels during thymopoiesis, with peak expression occurring at the double-positive thymocyte stage, and falling upon maturation and migration of the $\mathrm{T}$ cells to the periphery. They designed a vector carrying a conalbumin-specific TCR in which the vector transcript was made sensitive to suppression by endogenous miR-181a by inclusion of repetitive miR-181a target sequences following the transgene. In a murine model of HSC transplant to B10.D2 mice, known to negatively select this specific TCR, only transplants containing the TCR vector with the miR-181a target sequences resulted in the development of engineered mature TCR-expressing T cells that survived through thymic selection. Importantly, peripheral T cells expressing the conalbumin-specific TCR expanded when the animals were vaccinated with antigen-pulsed dendritic cells, and ex vivo functional assays showed responsiveness in an antigen dose-dependent fashion. This latter point demonstrates the feasibility for use of this approach for other potentially self-reactive TCRs (or CARs) in the clinical setting, as modulation of the TCR through development allowed self-reactive T-cell development yet did not ablate mature cell function. It would be of interest to know if the downregulation of TCR expression coincident with high levels of miR-181a during development will abrogate allelic exclusion and allow endogenous TCR rearrangement to occur, thereby permitting potential TCR mis-pairing in T cells expressing endogenous and exogenous TCR genes.

TCRs have the advantage over CARs of being able to recognize a wider variety of tumorassociated antigens, not limited to those that are expressed on the cell surface. Thus, the use 
of HSCs as a vehicle to continuously produce fresh TCR-modified T cells may provide an additional means to extend the activity of this approach.

\section{Driving CARs into HSCs}

As with gene modification of HSCs with TCRs, modification of HSCs with genes encoding CARs brings the prospect of long-term production of immune effector cells targeted to tumor-associated antigens. One major potential advantage with CARs is that their expression will not be limited to T cells, as is the case with TCR genes introduced into HSCs, since the surface display of CARs does not require CD3 co-expression. Thus, CARs may be expressed on multiple hematopoietic lineages ( $\mathrm{T}, \mathrm{NK}$, and myeloid), amplifying the potential graft-versus-cancer activity $(21,42-45)$. While de novo production of T cells from transplanted HSCs may be slow and limited in adult recipients, myeloid and NK cells should be produced rapidly after transplant, yielding an early source of anti-tumor immunity (4244). It is expected that CAR-bearing T cells would be the most efficient cytotoxic effectors cells against tumor cells produced by CAR-modified HSCs, due to their longer lifespan, greater activation and proliferation properties, and potential for generation of immunological memory. Results from studies assessing CAR-modified NK and myeloid cells indicate that NK cells have similar antigen-specific cytotoxicity to T cells $(42,43,46)$. However, the continued generation of large numbers of CAR-bearing neutrophils, monocytes and NK cells may constitute a powerful anti-cancer barrier, thanks to their tissue homing, large circulating pool in peripheral blood, and vascular diapedesis. Myeloid and NK cells do not depend on thymopoiesis to become active cytotoxic effectors and will exponentially amplify the anti-tumor immune response directed by CAR. The multi-lineage expression of CARs, associated with potent engineered antigen-specific cytotoxicity, makes the CAR modification of $\mathrm{HSC}$ a very promising cancer immunotherapy approach to be explored.

Furthermore, CAR-modified HSCs can be infused in the context of the standard medical procedure of hematopoietic stem cell transplantation (HSCT), when the chemotherapy conditioning regimen would create optimal conditions for the efficacy of this approach, favoring engraftment of gene-modified cells, as well as decreasing tumor burden and decreasing the risks of immunogenicity of the CAR molecules. Recently, a clinical trial has been opened infusing CD19-specific CAR-modified T cells in patients with high-risk Blineage malignancies undergoing autologous HSCT using UCB, to add potential graftversus-leukemia effects to the relatively naive $T$ cells contained within the graft (47). In this setting, CAR modification of a portion of the donor HSC may add additional anti-leukemic effects.

Studies performed by the research group at Cell Genesys, Inc. in the mid-1990s were the first efforts to explore the potential for using HSCs as the cell platform for expressing a CAR-like molecule $(42,43)$. They produced a CAR-like fusion protein between the extracellular domain of human CD4 and the intracellular CD3 $\zeta$ chain, termed a universal receptor (UR), to direct cytolytic effector cells to kill cells infected with HIV-1 through binding of the CD4 domain with HIV-1 gp120 displayed on the surface of the HIV-infected cell. This UR had been shown to arm T cells to kill HIV-infected cells and was advanced to several clinical trials into peripheral blood T cells, where it showed some evidence of in vivo anti-HIV effects $(42,48,49)$.

Moving towards a stem cell approach, this same investigative group studied the activity of the UR introduced into murine bone marrow HSCs, which was then transplanted into recipient mice. They demonstrated that it led to production of effective cytolytic cells, using a leukemia cell line transduced to express HIV-1 gp120 as a surrogate target for the CD4/ CD3 $\zeta$ fusion construct. Expression of the UR was observed on leukocytes of multiple 
lineages derived from the transduced HSCs, including granulocytes, monocytes, and NK cells. A direct demonstration that non-T cell effectors (myeloid and NK cells) were responsible for the cytolytic activity was obtained by transducing the UR gene into bone marrow from SCID mice (that would be genetically incapable of producing T lymphocytes), which still led to elimination of the gp120-expressing target cells in vivo after transplant (42). They also reported introduction of the UR into human blood NK cells and into human $\mathrm{CD} 4^{+}$cells that produced CAR-targeted myeloid cells upon in vitro differentiation and again demonstrated specific cytolytic activity (43).

In a later paper, these authors showed the insertion of the same $\mathrm{CD} 4 / \mathrm{CD} 3 \zeta$ fusion gene into marrow HSCs from normal mice led to production of UR-expressing myeloid, NK, and B cells, but a complete absence of transgenic T cells, with a blockade to production of URexpressing $\mathrm{T}$ cells occurring during the $\mathrm{T} 1 \rightarrow \mathrm{T} 2$ transition of thymopoiesis. This observation raises the specter that intrathymic negative selection of cells expressing a self-reactive CAR or TCR may prevent their maturation to mature T cells. However, the finding may be specific to this UR with a CD4 extracellular domain, because the UR-expressing T cells were not eliminated when the transduced HSC were transplanted into MHCII gene knockout mice, suggesting that it was a specific interaction between murine MHCII and human CD4 that triggered their elimination (50).

In exploring the role of the intracellular signaling domain, they also compared the CD3 $\zeta$ domain, used for TCR signaling in T cells, to the analogous domain from the $\mathrm{Fc}$ receptor- $\gamma$

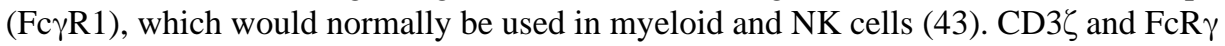
chains share the immunoreceptor tyrosine-based activation motif (ITAM), and both trigger activation pathways leading to target cytolysis, phagocytosis, and effector cell degranulation. In both murine and human T, myeloid, and NK cells, the UR with the CD3 $\zeta$ domain consistently displayed greater activity than one with the FcR $\gamma$ domain, which may be attributed to the presence of three ITAMs in the CD3 $\zeta$ domain but only one in the FcR $\gamma$ domain $(42,43)$. Other studies have reported similar findings on the greater activity of the $\mathrm{CD} 3 \zeta$ domain over the $\mathrm{FcR} \gamma$ domain in $\mathrm{T}$ cells $(42,43,51-54)$.

The optimal intracellular signaling domains for CAR to be used via HSCs are not fully known. The development of CAR activating moieties have mostly focused on the activation of $\mathrm{T}$ cells, with $\mathrm{CD} 3 \zeta$ being the most commonly used moiety $(51,55)$, as the terminal portion of the CAR construct, by itself (first generation CAR) or following the inclusion of different co-stimulatory domains (second and third generation CARs) $(56,57)$. Some knowledge has been obtained on the molecular events of the T-cell activation triggered by CARs, generally observing that the same activation pathways activated by TCR stimulation are involved. It has been demonstrated that CARs in T cells leads to immunological synapse formation $(58,59)$, calcium influx, and activation of the phosphatidylinositol and tyrosine kinase pathways $(60,61)$.

Although it is generally agreed that costimulation is required for efficacy in cancer immunotherapy approaches $(4,47,56,62,63)$, to this moment it is still not fully resolved which costimulatory molecule produces the best results in CAR-mediated T-cell activation (nor for myeloid and NK cells). It has been shown that CAR signaling mediates cell activation in neutrophils, monocytes, and NK cells, besides T cells, with either the FcR $\gamma$ or CD3 $\zeta$ activation moieties $(42,43,46,64)$. Activation of NK cells has been shown to require costimulation, with successful antigen-specific activation only achieved with secondgeneration CAR $(46,64)$. It was found that the $41 \mathrm{BB}$ costimulatory domain was most effective when CARs were introduced into mature human NK cells (64-66). Our group and others have shown that first generation CARs successfully activated neutrophils and 
monocytes $(42,43)$, and their antigen-specific cytotoxicity did not differ from that triggered by second generation CARs (DeOliveira et al., manuscript in revision).

Gene modification of HSCs requires stable transgene integration, ideally delivering one transgene copy per HSC (21). We have consistently achieved 40-50\% of transduction efficiency of human HSCs with CARs at an average of 1-3 copies/cell using lentiviral vectors with internal enhancer/promoters from the MND LTR. Higher transduction rates may not be warranted, as it would not be desired to completely devote the immune system to mono-specific anti-tumor effector cells; fortunately, for the immunotherapeutic applications, such high level engraftment of gene-modified human HSCs has not been achieved in clinical trials for genetic diseases where complete gene correction of all engrafted HSCs may be clinically useful.

CAR-modified HSCs undergo normal differentiation and proliferation, and their cell progeny are morphologically and functionally normal (DeOliveira et al., manuscript in revision). We have successfully engrafted anti-CD19 CAR-modified human CD34 ${ }^{+} \mathrm{HSC}$ in NSG mice, with CAR-bearing cells detected in all hematopoietic lineages (Fig. 4) in bone marrow, spleen, and peripheral blood. Notably, the absence of human CD19+ $\mathrm{B}$ cells suggests active cytotoxicity by the anti-CD19 CAR expressing cells, as B lymphocytes are normally the most common human cells to develop in this model.

With this initial progress towards modification of HSCs with CARs, several questions remain unresolved. The relative benefits of adding the activity of CAR-armed myeloid and NK cell effectors to the T-cells' army remain to be determined. It also remains to be determined whether the presence of the CAR will have adverse effects on de novo T-cell production, by causing negative selection, whether the CAR will suppress endogenous TCR gene rearrangement by allelic exclusion, as has been shown with TCRs, and whether the diverse CAR-expressing leukocytes will cause unique toxicities. Ongoing studies in the NSG mice may address some of these questions and initial clinical trials may provide more answers.

\section{Suicide genes to eliminate TCR/CAR modified HSC grafts}

With the engineering of HSCs comes the risk of insertional oncogenesis, as has occurred in several clinical trials in which $\gamma$-retroviral vectors were used to transduce autologous HSCs that were transplanted for patients with primary immune deficiency diseases (67). The introduction of these retroviral vectors, containing long terminal repeats with strong promoter/enhancer elements, into the susceptible genome of HSCs is capable of activating proximal cellular proto-oncogenes, and inducing cell proliferation that can progress to frank transformation. While the potential for this to occur in the setting of adult patients who would likely not have the high level of engraftment and proliferation seen in young immune deficiency patients, it remains a hypothetical risk. The long-term production of targeted effector cells, an attractive benefit of using HSC for immunotherapy, also poses potential problems if there is toxicity, either on-target/off-tissue toxicity, such as sustained suppression of $\mathrm{CD} 19^{+} \mathrm{B}$ cells, or the previously mentioned off-target reactivity possible by the introduction of a self-reactive TCR.

To alleviate these risks, the inclusion of a suicide gene to ablate gene-modified cells has been undertaken in a number of studies. The most widely studied suicide gene is derived from the herpes simplex virus thymidine kinase (HSV-TK) gene, which confers sensitivity to the anti-viral nucleoside analogue ganciclovir (68-73). Other suicide genes have been produced using fusion proteins that express FAS signaling moieties or caspase components under the post-transcriptional control of small molecule ligand (74-77). These latter genes 
are attractive in that they do not carry the immunogenicity demonstrated by the sr39TK construct, though they do not allow PET functionality to visualize modified cell tracking. The presence of a suicide gene in the TCR or CAR vector would allow cells that are overproliferating or causing toxicity to be eliminated by administration of the pro-drug or inducing agent.

In our studies using the NY-ESO-1 TCR lentiviral vector also carrying the sr39TK gene (described above), we examined its potential as a suicide gene to eliminate the cell products of the TCR-transduced human HSCs in the NSG mice. Upon administration of ganciclovir $(50 \mathrm{mg} / \mathrm{kg} \mathrm{IP} \times 5$ days $)$, the transduced cells were completely eliminated from the mice, as indicated by both repeat PET scans, FACS analysis for NY-ESO-1 tetramer staining, and PCR of blood, spleen, thymus, and bone marrow for the lentiviral psi element (not shown). The ability to eliminate the engineered graft is attractive in case of a serious adverse event, and remains an important safety switch for inclusion in HSC-based approaches to engineered immunity.

\section{Clinical considerations for use of TCR/CAR-modified HSCs}

Sustained HSC engraftment requires pre-transplant conditioning with agents that have myeloablative or at least marrow cytoreductive activity, such as total body irradiation or busulfan. These may be used at sub-myeloablative doses (e.g. $200 \mathrm{cGy}$ TBI or $4 \mathrm{mg} / \mathrm{kg}$ busulfan) for marrow cytoreduction with minimal acute toxicity. In contrast, the lymphodepleting agents typically used prior to T-cell immunotherapy (e.g. fludarabine, cyclophosphamide) are not significantly myeloablative and would not be expected to increase HSC engraftment. The need for cytoreductive conditioning as preparation for HSC immunotherapy would make application in disease settings where autologous or allogeneic HSCT is already used (e.g. leukemia and lymphoma) as most logical first choices.

The clinical application of TCR or CAR therapies via HSCT holds several challenges. Adult HSCs are generally more myeloid skewed than those from younger people, which may limit the numbers of T cells that can be produced from the transplanted, engineered HSCs (78). Additionally, the adult thymus is certainly much less active, undergoing involution with advancing age and the potential for de novo thymopoiesis is diminished, although some activity remains $(79,80)$. Prior treatments, such as chemotherapy or radiation therapy, may also decrease thymic function. These potential limits to new T-cell development emphasize the potential benefits derived from non-T-cell effectors that CAR-modified HSC may produce.

\section{Summary}

The potential advantages and proof-of-principles have been provided for approaches to cancer immunotherapy using TCR- or CAR-modified HSCs. It remains to be proven in clinical trials whether these approaches will lead to additive efficacy to those realized using mature $\mathrm{T}$ cells as targets. Certainly the two approaches may be complementary when applied together in some settings. Clinical trials are being developed that will provide some initial assessments.

\section{Acknowledgments}

Support was provided by the National Cancer Institute (1 PO1 CA132681), the Jonsson Comprehensive Cancer Center, and the Eli and Edythe Broad Center for Regenerative Medicine \& Stem Cell Research (D.B.K.); the UCLA Clinical and Translational Science Institute (UL1RR033176 and UL1TR000124), the UCLA Jonsson Comprehensive Cancer Center, and Hyundai Hope on Wheels Research Grant Award and St. Baldrick's Foundation Scholar Career Development Award \#180637 (S.N.O.); the California Institute for Regenerative Medicine Training Grant (TG2-01169) (E.G.). Antoni Ribas and Richard Koya (UCLA Department of Internal Medicine) provided the 
NY-ESO TCR-SR39 lentiviral vector plasmid. The Flow Cytometry Core of the Eli and Edythe Broad Center for Regenerative Medicine \& Stem Cell Research supported these studies. The authors have no conflicts of interest to declare.

\section{References}

1. Rosenberg SA, Spiess P, Lafreniere R. A new approach to the adoptive immunotherapy of cancer with tumor-infiltrating lymphocytes. Science. 1986; 233:1318-1321. [PubMed: 3489291]

2. Rosenberg SA, et al. Gene transfer into humans--immunotherapy of patients with advanced melanoma, using tumor-infiltrating lymphocytes modified by retroviral gene transduction. $\mathrm{N}$ Engl $\mathrm{J}$ Med. 1990; 323:570-578. [PubMed: 2381442]

3. Kalos M, Levine BL, Porter DL, Katz S, Grupp SA, Bagg A, June CH. T cells with chimeric antigen receptors have potent antitumor effects and can establish memory in patients with advanced leukemia. Sci Trans Med. 2011; 3:95ra73.

4. Porter DL, Levine BL, Kalos M, Bagg A, June CH. Chimeric antigen receptor-modified T cells in chronic lymphoid leukemia. N Engl J Med. 2011; 365:725-733. [PubMed: 21830940]

5. Grupp SA, et al. Chimeric antigen receptor-modified T cells for acute lymphoid leukemia. N Engl J Med. 2013; 368:1509-1518. [PubMed: 23527958]

6. Brentjens RJ, et al. CD19-targeted T cells rapidly induce molecular remissions in adults with chemotherapy-refractory acute lymphoblastic leukemia. Sci Trans Med. 2013; 5:177ra38.

7. Zhou Q, et al. Program death-1 signaling and regulatory T cells collaborate to resist the function of adoptively transferred cytotoxic T lymphocytes in advanced acute myeloid leukemia. Blood. 2010; 116:2484-2493. [PubMed: 20570856]

8. Ma C, et al. Multifunctional T-cell analyses to study response and progression in adoptive cell transfer immunotherapy. Cancer discovery. 2013; 3:418-429. [PubMed: 23519018]

9. Gattinoni L, et al. Acquisition of full effector function in vitro paradoxically impairs the in vivo antitumor efficacy of adoptively transferred CD8+ T cells. J Clin Invest. 2005; 115:1616-1626. [PubMed: 15931392]

10. Berger C, Jensen MC, Lansdorp PM, Gough M, Elliott C, Riddell SR. Adoptive transfer of effector CD8+ T cells derived from central memory cells establishes persistent $\mathrm{T}$ cell memory in primates. J Clin Invest. 2008; 118:294-305. [PubMed: 18060041]

11. Gattinoni L, Restifo NP. Moving T memory stem cells to the clinic. Blood. 2013; 121:567-568. [PubMed: 23349370]

12. Scholler J, et al. Decade-long safety and function of retroviral-modified chimeric antigen receptor T cells. Sci Trans Med. 2012; 4:132ra53.

13. Davila ML, Kloss CC, Gunset G, Sadelain M. CD19 CAR-targeted T cells induce long-term remission and B Cell Aplasia in an immunocompetent mouse model of B cell acute lymphoblastic leukemia. PloS One. 2013; 8:e61338. [PubMed: 23585892]

14. Bendle GM, et al. Lethal graft-versus-host disease in mouse models of $\mathrm{T}$ cell receptor gene therapy. Nat Med. 2010; 16:565-70. 1p following 570. [PubMed: 20400962]

15. Rosenberg SA. Of mice, not men: no evidence for graft-versus-host disease in humans receiving Tcell receptor-transduced autologous T cells. Mol Ther. 2010; 18:1744-1745. [PubMed: 20885433]

16. Schodin BA, Tsomides TJ, Kranz DM. Correlation between the number of T cell receptors required for T cell activation and TCR-ligand affinity. Immunity. 1996; 5:137-146. [PubMed: 8769477]

17. Nauerth M, et al. TCR-Ligand koff Rate Correlates with the Protective Capacity of AntigenSpecific CD8+ T Cells for Adoptive Transfer. Sci Trans Med. 2013; 5:192ra87-192ra87.

18. Schmitt TM, Ragnarsson GB, Greenberg P. T cell receptor gene therapy for cancer. Hum Gene Ther. 2009; 20:1240-1248. [PubMed: 19702439]

19. Notta F, Doulatov S, Laurenti E, Poeppl A, Jurisica I, Dick JE. Isolation of single human hematopoietic stem cells capable of long-term multilineage engraftment. Science. 2011; 333:218221. [PubMed: 21737740]

20. Doulatov S, Notta F, Laurenti E, Dick JE. Hematopoiesis: a human perspective. Cell Stem Cell. 2012; 10:120-136. [PubMed: 22305562] 
21. Kohn DB, Pai S-Y, Sadelain M. Gene therapy through autologous transplantation of gene-modified hematopoietic stem cells. Biol Blood Marrow Transplant. 2013; 19:S64-S69. [PubMed: 23032601]

22. Giannoni F, et al. Allelic exclusion and peripheral reconstitution by TCR transgenic T cells arising from transduced human hematopoietic stem/progenitor cells. Mol Ther. 2013; 21:1044-54. [PubMed: 23380815]

23. Dudley ME, et al. Adoptive cell therapy for patients with metastatic melanoma: evaluation of intensive myeloablative chemoradiation preparative regimens. J Clin Oncol. 2008; 26:5233-5239. [PubMed: 18809613]

24. Pogulis RJ, Hansen MJ, Pease LR. Retroviral-mediated expression of an MHC class I-restricted T cell receptor in the CD8 T cell compartment of bone marrow-reconstituted mice. Hum Gene Ther. 1998; 9:2285-2297. [PubMed: 9794212]

25. Clay TM, Custer MC, Spiess PJ, Nishimura MI. Potential use of T cell receptor genes to modify hematopoietic stem cells for the gene therapy of cancer. Pathol Oncol Res. 1999; 5:3-15. [PubMed: 10079371]

26. Yang L, Baltimore D. Long-term in vivo provision of antigen-specific T cell immunity by programming hematopoietic stem cells. Proc Natl Acad Sci USA. 2005; 102:4518-4523. [PubMed: 15758071]

27. Ha SP, Klemen ND, Kinnebrew GH, Brandmaier AG, Marsh J, Hangoc G, Palmer DC, et al. Transplantation of mouse HSCs genetically modified to express a CD4-restricted TCR results in long-term immunity that destroys tumors and initiates spontaneous autoimmunity. J Clin Invest. 2010; 120:4273-88. [PubMed: 21084750]

28. Alajez NM, Schmielau J, Alter MD, Cascio M, Finn OJ. Therapeutic potential of a tumor-specific, MHC-unrestricted T-cell receptor expressed on effector cells of the innate and the adaptive immune system through bone marrow transduction and immune reconstitution. Blood. 2005; 105:4583-4589. [PubMed: 15746083]

29. Zhao Y, et al. Extrathymic generation of tumor-specific T cells from genetically engineered human hematopoietic stem cells via Notch signaling. Cancer Res. 2007; 67:2425-2429. [PubMed: 17363559]

30. van Lent AU, et al. Functional human antigen-specific $\mathrm{T}$ cells produced in vitro using retroviral $\mathrm{T}$ cell receptor transfer into hematopoietic progenitors. J Immunol. 2007; 179:4959-4968. [PubMed: 17911580]

31. Kitchen SG, et al. Engineering antigen-specific T cells from genetically modified human hematopoietic stem cells in immunodeficient mice. PloS One. 2009; 4:e8208. [PubMed: 19997617]

32. Vatakis DN, et al. Antitumor activity from antigen-specific CD8 T cells generated in vivo from genetically engineered human hematopoietic stem cells. Proc Natl Acad Sci USA. 2011; 108:E1408-E1416. [PubMed: 22123951]

33. Greenblatt MB, Vbranac V, Tivey T, Tsang K, Tager AM, Aliprantis AO. Graft versus host disease in the bone marrow, liver and thymus humanized mouse model. PloS One. 2012; 7:e44664. [PubMed: 22957096]

34. Shultz LD, et al. Generation of functional human T-cell subsets with HLA-restricted immune responses in HLA class I expressing NOD/SCID/IL2r gamma(null) humanized mice. Proc Natl Acad Sci USA. 2010; 107:13022-13027. [PubMed: 20615947]

35. Mostoslavsky R, Alt FW, Rajewsky K. The lingering enigma of the allelic exclusion mechanism. Cell. 2004; 118:539-544. [PubMed: 15339659]

36. Vatakis DN, Arumugam B, Kim SG, Bristol G, Yang O, Zack JA. Introduction of exogenous Tcell receptors into human hematopoietic progenitors results in exclusion of endogenous T-cell receptor expression. Mol Ther. 2013; 21:1055-1063. [PubMed: 23481324]

37. Wargo JA, et al. Recognition of NY-ESO-1+ tumor cells by engineered lymphocytes is enhanced by improved vector design and epigenetic modulation of tumor antigen expression. Cancer Immunol Immunother. 2009; 58:383-394. [PubMed: 18677478] 
38. Black ME, Kokoris MS, Sabo P. Herpes simplex virus-1 thymidine kinase mutants created by semi-random sequence mutagenesis improve prodrug-mediated tumor cell killing. Cancer Res. 2001; 61:3022-3026. [PubMed: 11306482]

39. Cheever MA, et al. The prioritization of cancer antigens: a national cancer institute pilot project for the acceleration of translational research. Clin Cancer Res. 2009; 15:5323-5337. [PubMed: 19723653]

40. Deng L, Mariuzza RA. Recognition of self-peptide-MHC complexes by autoimmune T-cell receptors. Trends Biochem Sci. 2007; 32:500-508. [PubMed: 17950605]

41. Papapetrou EP, Kovalovsky D, Beloeil L, Sant'angelo D, Sadelain M. Harnessing endogenous miR-181a to segregate transgenic antigen receptor expression in developing versus post-thymic $\mathrm{T}$ cells in murine hematopoietic chimeras. J Clin Invest. 2009; 119:157-168. [PubMed: 19033646]

42. Hege KM, Cooke KS, Finer MH, Zsebo KM, Roberts MR. Systemic T cell-independent tumor immunity after transplantation of universal receptor-modified bone marrow into SCID mice. J Exp Med. 1996; 184:2261-2269. [PubMed: 8976181]

43. Roberts MR, et al. Antigen-specific cytolysis by neutrophils and NK cells expressing chimeric immune receptors bearing zeta or gamma signaling domains. J Immunol. 1998; 161:375-384. [PubMed: 9647246]

44. Badowski MS, Zhang T, Tsang TC, Harris DT. Chimeric antigen receptors for stem cell based immunotherapy. J Exp Ther Oncol. 2009; 8:53-63. [PubMed: 19827271]

45. Doering CB, Archer D, Spencer HT. Delivery of nucleic acid therapeutics by genetically engineered hematopoietic stem cells. Adv Drug Delivery Rev. 2010; 62:1204-1212.

46. Tran AC, Zhang D, Byrn R, Roberts MR. Chimeric zeta-receptors direct human natural killer (NK) effector function to permit killing of NK-resistant tumor cells and HIV-infected T lymphocytes. J Immunol. 1995; 155:1000-1009. [PubMed: 7608531]

47. Kebriaei P, et al. Infusing CD19-directed T cells to augment disease control in patients undergoing autologous hematopoietic stem-cell transplantation for advanced B-lymphoid malignancies. Hum Gene Ther. 2012; 23:444-450. [PubMed: 22107246]

48. Walker RE, et al. Long-term in vivo survival of receptor-modified syngeneic $\mathrm{T}$ cells in patients with human immunodeficiency virus infection. Blood. 2000; 96:467-474. [PubMed: 10887107]

49. Mitsuyasu RT, et al. Prolonged survival and tissue trafficking following adoptive transfer of CD4zeta gene-modified autologous CD4(+) and CD8(+) T cells in human immunodeficiency virus-infected subjects. Blood. 2000; 96:785-793. [PubMed: 10910888]

50. Lin WY, Roberts MR. Developmental dissociation of T cells from B, NK, and myeloid cells revealed by MHC class II-specific chimeric immune receptors bearing TCR-zeta or FcR-gamma chain signaling domains. Blood. 2002; 100:3045-3048. [PubMed: 12351421]

51. Letourneur F, Klausner RD. T-cell and basophil activation through the cytoplasmic tail of T-cellreceptor zeta family proteins. Proc Natl Acad Sci USA. 1991; 88:8905-8909. [PubMed: 1833767]

52. Eshhar Z, Waks T, Gross G, Schindler DG. Specific activation and targeting of cytotoxic lymphocytes through chimeric single chains consisting of antibody-binding domains and the gamma or zeta subunits of the immunoglobulin and T-cell receptors. Proc Natl Acad Sci USA. 1993; 90:720-724. [PubMed: 8421711]

53. Darcy PK, et al. Redirected perforin-dependent lysis of colon carcinoma by ex vivo genetically engineered CTL. J Immunol. 2000; 164:3705-3712. [PubMed: 10725729]

54. Haynes NM, et al. Redirecting mouse CTL against colon carcinoma: superior signaling efficacy of single-chain variable domain chimeras containing TCR-zeta vs Fc epsilon RI-gamma. J Immunol. 2001; 166:182-187. [PubMed: 11123291]

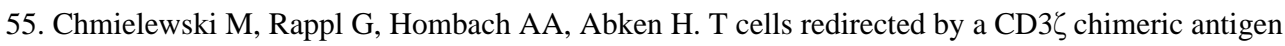
receptor can establish self-antigen-specific tumour protection in the long term. Gene Ther. 2013; 20:177-186. [PubMed: 22378346]

56. Kohn DB, et al. CARs on track in the clinic. Molecular therapy. 2011; 19:432-438. [PubMed: 21358705]

57. Sadelain M, Brentjens R, Riviére I. The basic principles of chimeric antigen receptor design. Cancer Disc. 2013; 3:388-398. 
58. Bridgeman JS, Hawkins RE, Bagley S, Blaylock M, Holland M, Gilham DE. The optimal antigen response of chimeric antigen receptors harboring the $\mathrm{CD} 3$ zeta transmembrane domain is dependent upon incorporation of the receptor into the endogenous TCR/CD3 complex. J Immunol. 2010; 184:6938-6949. [PubMed: 20483753]

59. Rappl G, et al. The CD3-zeta chimeric antigen receptor overcomes TCR Hypo-responsiveness of human terminal late-stage T cells. PloS One. 2012; 7:e30713. [PubMed: 22292024]

60. Romeo C, Amiot M, Seed B. Sequence requirements for induction of cytolysis by the T cell antigen/Fc receptor zeta chain. Cell. 1992; 68:889-897. [PubMed: 1547489]

61. Zhong X-S, Matsushita M, Plotkin J, Riviere I, Sadelain M. Chimeric antigen receptors combining 4-1BB and CD28 signaling domains augment PI3kinase/AKT/Bcl-XL activation and CD8+ T cellmediated tumor eradication. Mol Ther. 2010; 18:413-420. [PubMed: 19773745]

62. Kochenderfer JN, et al. Eradication of B-lineage cells and regression of lymphoma in a patient treated with autologous T cells genetically engineered to recognize Brief report Eradication of Blineage cells and regression of lymphoma in a patient treated with autologous T cells. Blood. 2010; 116:4099-4102. [PubMed: 20668228]

63. Savoldo B, et al. CD28 costimulation improves expansion and persistence of chimeric antigen receptor-modified T cells in lymphoma patients. The Journal of clinical investigation Clin Invest. 2011; 121:1822-1826.

64. Shook DR, Campana D. Natural killer cell engineering for cellular therapy of cancer. Tissue Antigens. 2011; 78:409-415. [PubMed: 22077621]

65. Milone MC, et al. Chimeric receptors containing CD137 signal transduction domains mediate enhanced survival of T cells and increased antileukemic efficacy in vivo. Mol Ther. 2009; 17:1453-1364. [PubMed: 19384291]

66. Li L, et al. Expression of chimeric antigen receptors in natural killer cells with a regulatorycompliant non-viral method. Cancer Gene Ther. 2010; 17:147-154. [PubMed: 19745843]

67. Mukherjee S, Thrasher AJ. Gene therapy for PIDs: Progress, pitfalls and prospects. Gene. 2013; 525:174-181. [PubMed: 23566838]

68. Bonini C, et al. HSV-TK gene transfer into donor lymphocytes for control of allogeneic graftversus-leukemia. Science. 1997; 276:1719-1724. [PubMed: 9180086]

69. Riddell SR, et al. T-cell mediated rejection of gene-modified HIV-specific cytotoxic T lymphocytes in HIV-infected patients. Nat Med. 1996; 2:216-223. [PubMed: 8574968]

70. Berger C, Flowers ME, Warren EH, Riddell SR. Analysis of transgene-specific immune responses that limit the in vivo persistence of adoptively transferred HSV-TK-modified donor T cells after allogeneic hematopoietic cell transplantation. Blood. 2006; 107:2294-2302. [PubMed: 16282341]

71. Traversari C, et al. The potential immunogenicity of the TK suicide gene does not prevent full clinical benefit associated with the use of TK-transduced donor lymphocytes in HSCT for hematologic malignancies. Blood. 2007; 109:4708-4715. [PubMed: 17327417]

72. Morris JC, Conerly M, Thomasson B, Storek J, Riddell SR, Kiem H-P. Induction of cytotoxic Tlymphocyte responses to enhanced green and yellow fluorescent proteins after myeloablative conditioning. Blood. 2004; 103:492-499. [PubMed: 14512305]

73. Arber $\mathrm{C}$, et al. The immunogenicity of virus-derived $2 \mathrm{~A}$ sequences in immunocot mpetent individuals. Gene Ther. 2013; 20:958-962. [PubMed: 23698740]

74. MacCorkle RA, Freeman KW, Spencer DM. Synthetic activation of caspases: artificial death switches. Proc Natl Acad Sci USA. 1998; 95:3655-3660. [PubMed: 9520421]

75. Clackson T, et al. Redesigning an FKBP-ligand interface to generate chemical dimerizers with novel specificity. Proc Natl Acad Sci USA. 1998; 95:10437-10442. [PubMed: 9724721]

76. Thomis DC, et al. A Fas-based suicide switch in human T cells for the treatment of graft-versushost disease. Blood. 2001; 97:1249-1257. [PubMed: 11222367]

77. Straathof KC, et al. An inducible caspase 9 safety switch for T-cell therapy. Blood. 2005; 105:4247-4254. [PubMed: 15728125]

78. Benz C, et al. Hematopoietic stem cell subtypes expand differentially during development and display distinct lymphopoietic programs. Cell Stem Cell. 2012; 10:273-283. [PubMed: 22385655]

79. Mackall CL, et al. Age, thymopoiesis, and CD4+ T-lymphocyte regeneration after intensive chemotherapy. N Engl J Med. 1995; 332:143-149. [PubMed: 7800006] 
80. Weinberg K, Annett G, Kashyap A, Lenarsky C, Forman SJ, Parkman R. The effect of thymic function on immunocompetence following bone marrow transplantation. Biol Blood Marrow Transplant. 1995; 1:18-23. [PubMed: 9118285] 


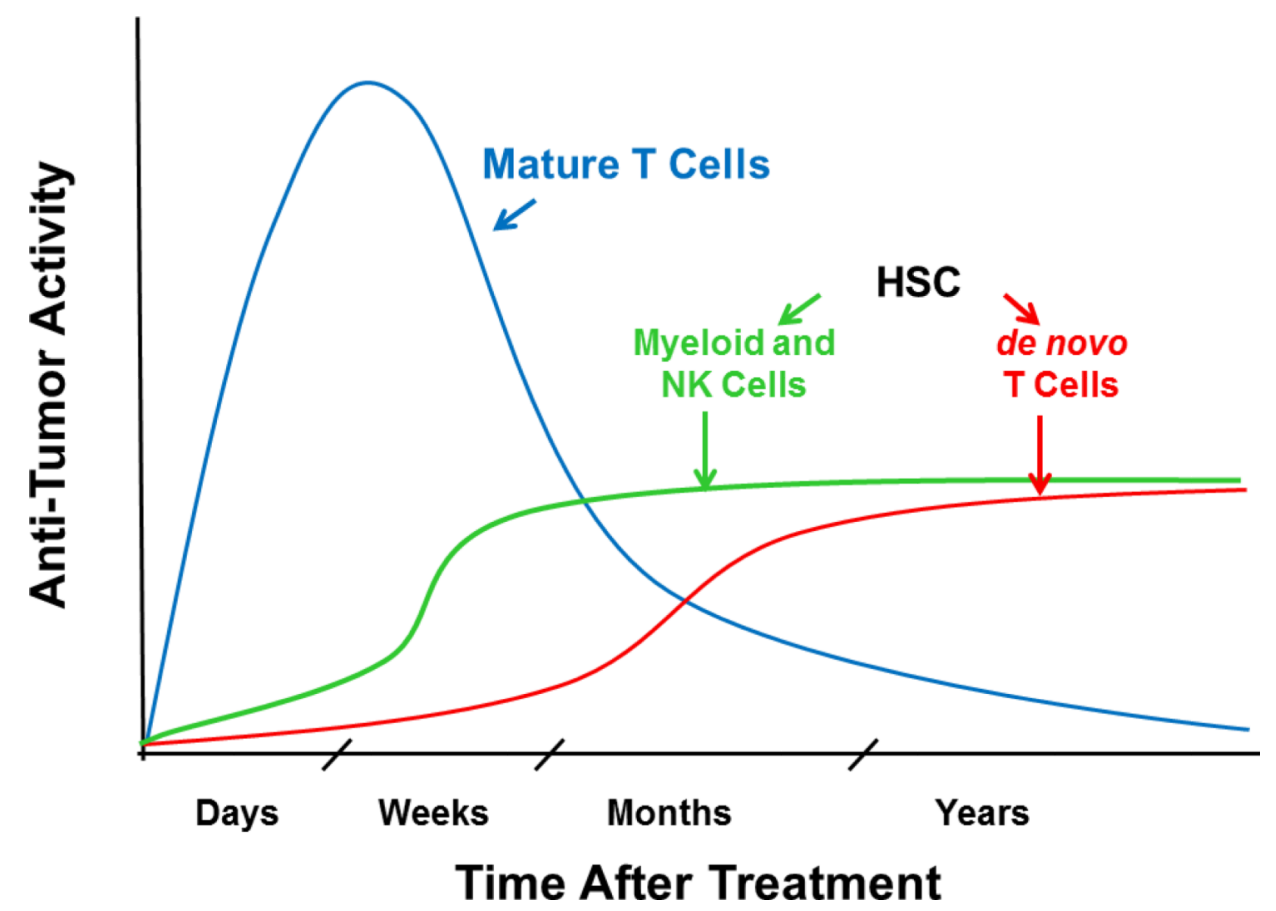

Fig. 1. Theoretical time course of anti-tumor activity

The theoretical anti-tumor activity produced in patients is depicted following a single infusion of TCR- or CAR-transduced mature T cells (blue), and following stable engraftment of TCR- or CAR-transduced HSCs, yielding myeloid and NK cells expressing CARs (green) within weeks of transplant and de novo $\mathrm{T}$ cells expressing TCRs or CARs (red) within months. 


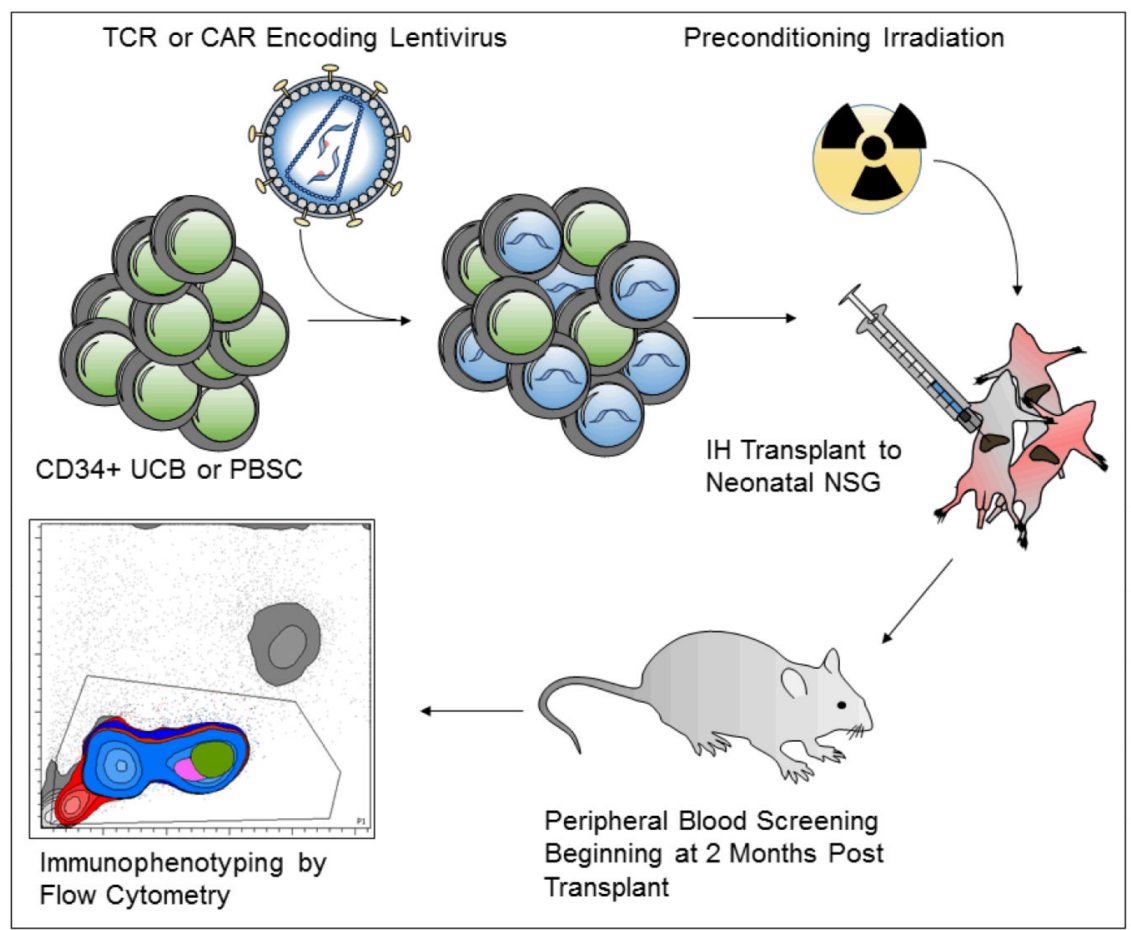

Fig. 2. In vivo xenograft model to study the use of hematopoietic stem cells for cancer immunotherapy

Umbilical cord blood (UCB) or peripheral blood stem cells (PBSCs) are processed to purify the $\mathrm{CD}_{34}{ }^{+}$population enriched for HSCs. Cells are pre-stimulated and transduced with a concentrated lentiviral vector encoding a TCR or CAR. Gene-modified cells are transplanted via intra-hepatic (IH) injection to pre-conditioned (150 cGy) NSG neonatal mice. Beginning at 2 months post-transplantation, immunophenotypic analysis of peripheral blood is performed by polychromatic flow cytometry. 

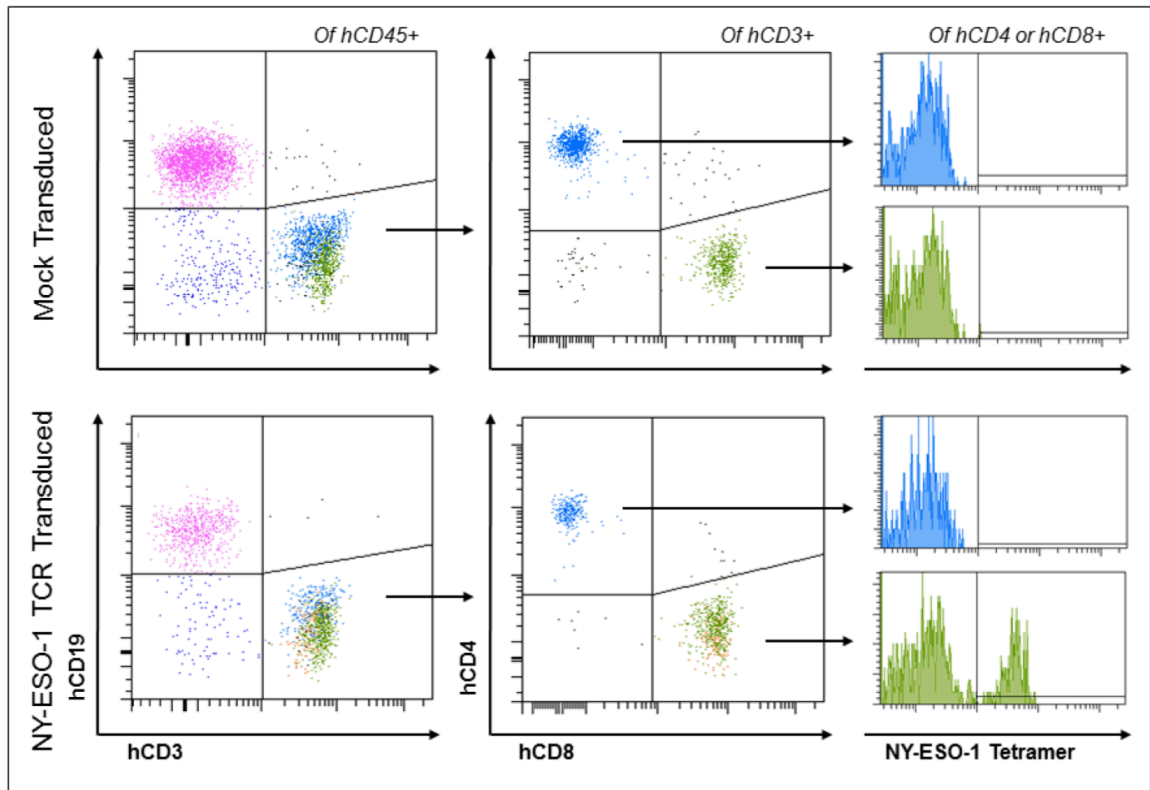

Fig. 3. Immunophenotypic analysis of humanized mice

NSG mice transplanted with either mock-transduced and NY-ESO-1-TCR vector-transduced PBSC reconstitute a human immune system in vivo, as evidenced by human $\mathrm{CD} 19^{+} \mathrm{B}$ cells and both $\mathrm{CD}^{+}$and $\mathrm{CD}^{+}$human $\mathrm{CD}^{+}{ }^{+} \mathrm{T}$ cells. $\mathrm{CD}{ }^{+}, \mathrm{NY}-\mathrm{ESO}-1$ tetramer-binding effector $\mathrm{T}$ cells that developed from gene-modified HSCs are detectable (bottom right). 

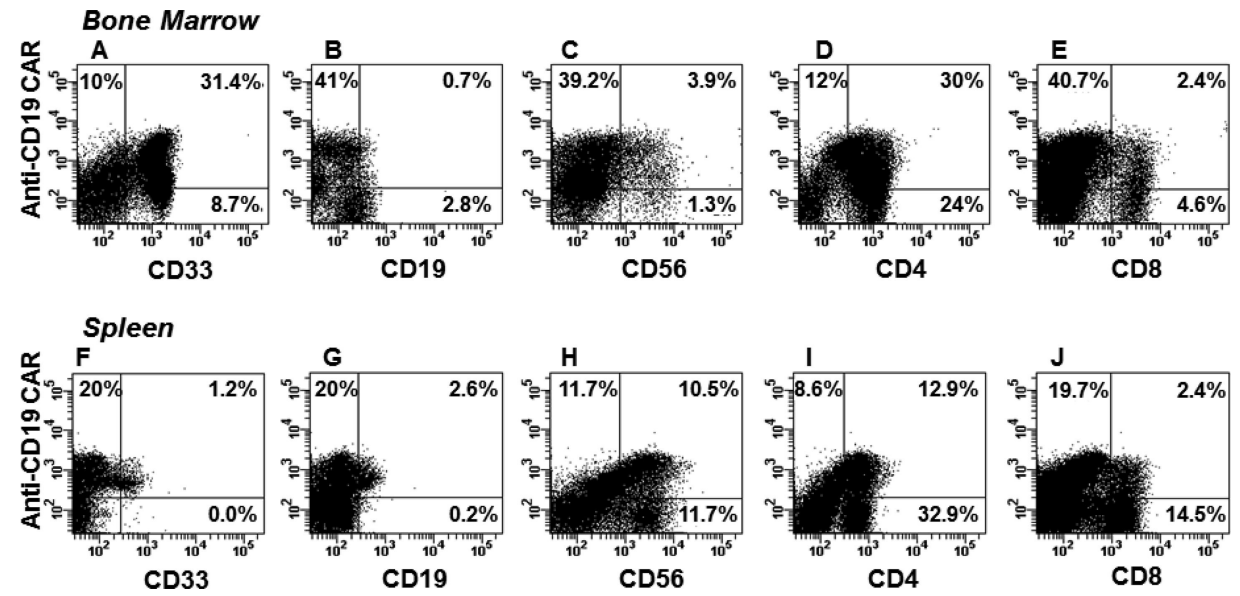

Fig. 4. CD19-specific CAR-bearing cells in humanized NSG mouse

Representative flow cytometry dot plots from a humanized mouse engrafted with CARmodified human HSCs are depicted. (A-E). Cells from bone marrow were analyzed after staining with a FITC-labeled anti-IgG Fc to detect CAR (Y axis) versus antibodies against the human surface markers CD33 (myeloid cell marker), CD19 (B-cell marker), CD56 (NK cell marker), CD4 and CD8 (T-cell markers), represented on X axis. (F-J). Cells from spleen of the same mouse stained using same antibody mix. Bold numbers in quadrants represent percentages from total human CD45 population in each sample. 